\title{
Mitigation of preferential concentration of small inertial particles in stationary isotropic turbulence using electrical and gravitational body forces.
}

\author{
KARNIK, A.U. and SHRIMPTON, J.S.
}




\section{Mitigation of preferential concentration of small inertial particles in stationary isotropic turbulence using electrical and gravitational body forces}

Cite as: Phys. Fluids 24, 073301 (2012); https://doi.org/10.1063/1.4732540

Submitted: 28 October 2011 . Accepted: 31 May 2012 . Published Online: 16 July 2012

Aditya U. Karnik, and John S. Shrimpton

ARTICLES YOU MAY BE INTERESTED IN

Preferential concentration of particles by turbulence

Physics of Fluids A: Fluid Dynamics 3, 1169 (1991); https://doi.org/10.1063/1.858045

Charged particle dynamics in turbulence: Theory and direct numerical simulations

Physics of Fluids 27, 065111 (2015); https://doi.org/10.1063/1.4922645

Effect of preferential concentration on turbulent collision rates

Physics of Fluids 12, 2530 (2000); https://doi.org/10.1063/1.1288515

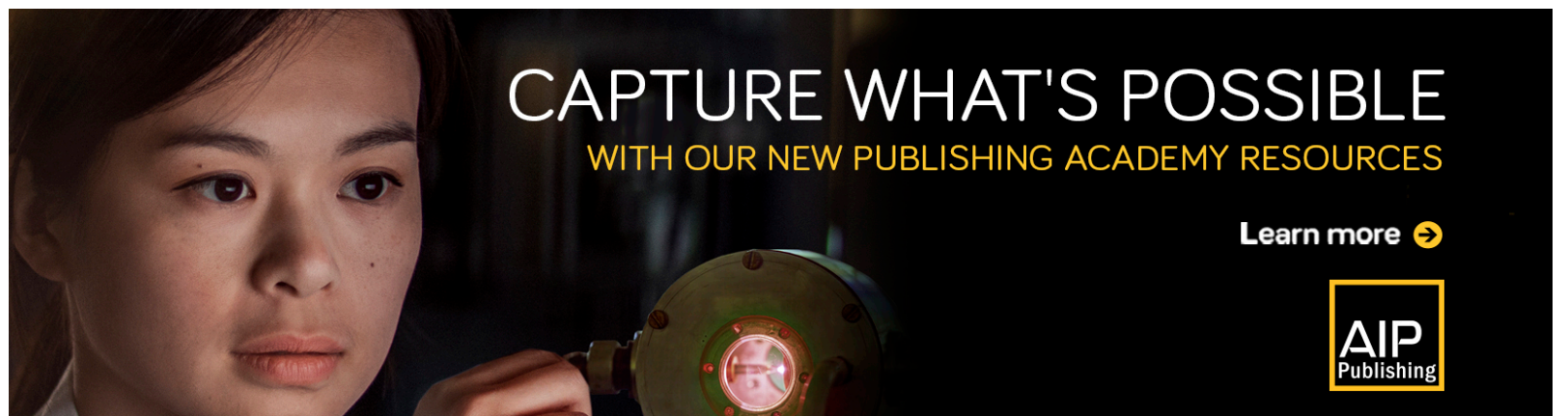




\title{
Mitigation of preferential concentration of small inertial particles in stationary isotropic turbulence using electrical and gravitational body forces
}

\author{
Aditya U. Karnik and John S. Shrimpton ${ }^{\text {a) }}$ \\ Energy Technology Research Group, School of Engineering Sciences, \\ University of Southampton, Southampton S017 1BJ, United Kingdom
}

(Received 28 October 2011; accepted 31 May 2012; published online 16 July 2012)

\begin{abstract}
Particles with a certain range of Stokes numbers preferentially concentrate due to action of turbulent motion and body forces such as gravity are known to influence this process. The effect of electric charge, residing on particles, upon the phenomenon of preferential concentration is investigated. We use direct numerical simulations of oneway coupled stationary isotropic turbulence over a range of particle Stokes numbers, fluid Taylor Reynolds numbers, and electrical and gravitational particle body force magnitudes, the latter characterized by non-dimensional settling velocities, $v_{c}^{*}$ and $v_{g}^{*}$, respectively. In contrast to the gravitational body force, the electrical analogue, acting on an electrically charged particle, is generated by an electric field, which is in turn a function of the degree of preferential concentration. Thus, the electrical body force is created by, and mitigates, preferential concentration. In the absence of gravity, it is estimated that $v_{c}^{*} \approx 1.0$ is sufficient to homogenise a preferentially concentrated particle distribution. It is seen that charging drastically reduces the radial distribution function values at Kolmogorov scale separations, which gravitational force does not. This implies that charging the particles is an efficient means to destroy small clusters of particles. On incorporating the gravitational force, the amount of charge required to homogenise the particle distribution is reduced. It is estimated that $v_{c}^{*} \approx 0.6$ is sufficient to homogenise particle distribution at $v_{g}^{*}=2.0$. This estimation is corroborated by several different indicators of preferential concentration, and the results also agree reasonably well with corresponding experiments reported in literature. Calculations also suggest that sprays generated by practical charge injection atomizers would benefit from this electrical dispersion effect. ( 2012 American Institute of Physics. [http://dx.doi.org/10.1063/1.4732540]
\end{abstract}

\section{INTRODUCTION}

This study focuses on dispersion of small heavy particles in a turbulent continuum affected by particle body forces using direct numerical simulation (DNS) of turbulence. DNS has been widely used to compute particle dispersion starting with the pioneering work of Riley and Patterson. ${ }^{1}$ Besides providing an accurate description of the instantaneous fluid velocity field, it also allows the detailed analysis of Eulerian and Lagrangian particle statistics, the latter extremely challenging to obtain experimentally. Of particular interest is the clustering of small heavy particles in certain regions of the flow, typically referred to as preferential concentration. Theoretical investigations of the effect of particle inertia on dispersion have been made by Yudine, ${ }^{2}$ Csanady, ${ }^{3}$ and Reeks ${ }^{4}$ amongst others. Csanady ${ }^{3}$ demonstrated that the rapid travel of heavy particles relative to the surrounding turbulent eddies could result in appreciable reduction of dispersion rates.

\footnotetext{
a) Author to whom correspondence should be addressed. Electronic mail: john.shrimpton@ soton.ac.uk. Telephone: +44[0]23-8059-4894. Fax: +44-[0]23-8059-3058.
} 
Here, we briefly review different aspects of preferential concentration phenomena and the applications where it plays a significant role. Wang and Maxey ${ }^{5}$ found that for particles with a certain response time there was a significant increase in the average settling velocity. Their numerical work demonstrated the significance of the Kolmogorov scaling to these processes. Elghobashi and Truesdell ${ }^{6}$ used DNS to calculate trajectories of particles and undertook a systematic investigation of effect of the inertia (drag force) and gravity (buoyancy force) on the dispersion statistics. The set of data provided by Elghobashi and Truesdell, ${ }^{6}$ combined with the data from the experiment of Snyder and Lumley $^{7}$ (which Ref. 6 tries to replicate) provides an excellent means to validate contemporary work on turbulent dispersion of solid particles and also the limitations of so called "DNS" implicit in the point particle assumption. Eaton and Fessler ${ }^{8}$ reviewed preferential concentration in a wide range of flows and identified centrifuging of particles away from vortex cores as the basic mechanism in most flows. Squires and Eaton ${ }^{9}$ showed preferential concentration in the convergence (or high-strain, low-vorticity) regions of the flow. However, recently it has been pointed out that clustering also takes place for particles with relaxation times larger than the Kolmogorov time scale. ${ }^{10}$ The role of multiscale eddies at higher Reynolds numbers in clustering at inertial scales has been demonstrated in 2D turbulence by Boffetta et al.,${ }^{11}$ Goto and Vassilicos ${ }^{12}$ and also in 3D. ${ }^{13}$

More recently the process of segregation of inertial particles has been studied from a more dynamical viewpoint, under the restriction that the Stokes number is small. Balkovsky et al. ${ }^{14}$ showed that the preferential concentration is highly intermittent and continues indefinitely. Dynamical systems theory has been applied to the motion of particles, first by Sommerer and Ott ${ }^{15}$ and in turbulent flows by $\mathrm{Bec}^{16}$ and Wilkinson et al. ${ }^{17}$ who showed that periods in time exist where particle trajectories may cross leading to particle collisions. Relaxing an assumption of Wilkinson et al. ${ }^{17}$ that the typical correlation time of the carrier flow is small, Reeks and co-workers have deployed the full Lagrangian method (FLM) of Ostiptsov ${ }^{18}$ first in synthetic flows ${ }^{19}$ and recently in isotropic turbulence ${ }^{20}$ to quantify non-uniformities and singularities in the spatial distribution of particles more generally. The advantage of FLM being that the analysis takes place on infinitesimally small scales and does not rely on defining a box size for counting purposes and that a small Stokes number limit is not implicit in the method. The research confirms that the particle concentration field is highly intermittent in nature and that the particle collision rate has an activation dependence on Stokes number. Higher Stokes numbers however present more challenges in understanding due to the increase in random uncorrelated motion of the particle velocity field ${ }^{21}$ and the memory large inertia particles have of their past motion across several turbulent eddies.

Traditionally, the $D_{c}$ and $D_{n}$ measures ${ }^{5}$ are used to quantify deviation of the particle concentration from a randomly defined distribution. In their experimental study, Fessler et $a l .{ }^{22}$ calculated the $D$ parameter to quantify deviation of local particle number density from that of a random distribution. Evaluation of the Eulerian $D_{c}, D_{n}, D$ measures involves the binning of the particles in cubic bins of size $h$ and hence the measures exhibit a dependence on the bin-size used. It is observed that these measures have a maxima for $h \approx 10 \eta$ (where $\eta$ is the Kolmogorov length scale) and this can be thought of as the characteristic length scale of the clusters. ${ }^{22,23}$ The radial distribution function (RDF) introduced by Sundaram and Collins ${ }^{24}$ and by used Collins and Keswani ${ }^{25}$ to account for effect of particle clustering on collision efficiency. The correlation dimension, $D_{2}$, is a measure of the $\mathrm{RDF}$ and has been used to quantify accumulation in a number of studies. ${ }^{26,27}$ Eulerian measures of preferential concentration such as $D_{c}, D_{n}, D$ tend to show a dependence on the turbulence intensity whereas Lagrangian measures, such as the RDF and $D_{2}$ do not. As outlined by Scott $e t$ al. ${ }^{28}$ Eulerian measures record cluster structure scales whereas the RDF and $D_{2}$ depend truly on the distribution of particle-particle spacing.

Whilst numerous investigations, both experimental and numerical, have demonstrated the phenomenon of preferential concentration, there has been much less emphasis, if any, focussed on its control. For applications such as combustion, accumulation of particles in vortex structures is a problem because of the in-homogenous fuel vapour-air mixture generated. The combustion process will not be efficient and may produce unwanted emissions. Indeed, Eaton and Fessler ${ }^{8}$ had noted that preferential concentration might play a significant role in a number of practical applications ranging from coal fired combustors to electrostatic precipitators. There is clearly a need, since several liquid fuelled combustion systems with great potential require highly homogenous combustion 
mixtures, two examples being pulse detonation engines ${ }^{29}$ and homogenous charge compression ignition engines. ${ }^{30}$ The issue is of more than academic interest since current technology, charged injection atomizers, are capable of generating electrically charged sprays of electrically insulating hydrocarbon liquids and have been developed and refined. ${ }^{31}$ Electrically charged sprays offer several benefits such as the lack of droplet agglomeration and control of the droplet size distribution and spray plume shape. ${ }^{32}$ This contribution explores one technique to significantly reduce preferential concentration that may be practically realizable.

For liquid fuel injection systems in combustion systems, the issue is that once the liquid primary atomization process has completed, control of the particle number density distribution downstream of the atomizer is lost, since the only means to modify the drop trajectory is via the mean and fluctuating velocity fields of the fluid. Given the possibility of de-mixing of particle population due to interaction with the smaller scales of the fluctuating velocity, the possibility of using the fluid velocity field to obtain a uniform particle concentration can be ruled out. Given the above, what is ideally required is an intelligent particle body force which switches itself "on" once the particle number density becomes spatially non-uniform. This is precisely what an electrical body force can in theory achieve. Only when spatial fluctuations in the particle concentration field exist is an electric field produced, with a direction to reduce the concentration inhomogeneity. Therefore, a link exists between preferential concentration-which creates an electric field, and the electric field-which acts to mitigate preferential concentration. In essence, there are three factors to consider, the particle space charge (a function of the particle charge and the bulk number density), the particle Stokes number (the propensity of a given particle to undergo preferential concentration, and thus create an electric field) and the fluid Taylor Reynolds number (where at higher values, more scales are involved in the preferential concentration process).

In the context of Diesel engine time scales and ambient conditions, the expansion rate of a spherical cloud of evaporating charged particles in a quiescent medium has been investigated by Bellan. ${ }^{33}$ Further work ${ }^{34}$ examined the dispersion of a cluster of charged drops in an inviscid vortex and showed that charging drops to $50 \%$ of the Rayleigh limit increases the dispersion within the vortex by a factor of 2 and decreases the evaporation time by $50 \%$. The effect of charge is to increase the drop velocity away from the vortex centre by a factor of nearly 10 and to reduce the drop number density. The reduction in fuel mass fraction reduces the tendency of soot production. Whilst these studies $^{33,34}$ revealed the potential effect of electric charge on the dispersion of a cluster of nearby drops, including beneficial effect of evaporation rate, ${ }^{34}$ they are restricted to highly simplified continuum velocity assumptions and do not account for the now well known particle-turbulence interactions leading to particle clustering.

Very few studies have addressed the clustering of charged particles. Alipchenkov et al. ${ }^{35}$ have suggested a statistical model to explain clustering of charged particles in isotropic turbulence. Lu et al. ${ }^{36}$ reported measurements of clustering of charged particles in isotropic turbulence. It has been observed that the RDF increases exponentially at sub-Kolmogorov scales for uncharged particles and this power law behaviour has been explained by Chun $e$ al. $^{37}$

$$
g(r)=C_{0}\left(\frac{\eta}{r}\right)^{C_{1}}
$$

where $\eta$ is the Kolmogorov length scale, $r$ the inter-particle distance, and $C_{0}$ is an unspecified matching coefficient whose value depends upon how the locally smooth flow transitions to turbulence at larger separations. $C_{l}$ is defined as

$$
C_{1}=3.61 S t_{k} \tau_{\eta}\left(\left\langle S^{2}\right\rangle-\left\langle R^{2}\right\rangle\right),
$$

where $S$ and $R$ are the second invariants of the rate of strain and rotation tensors, respectively, averaged over an ensemble of particle trajectories. $S t_{k}$ is the Stokes number, the ratio of the particle relaxation timescale $\tau_{p}$ to the Kolmogorov timescale $\tau_{\eta}$. The experimental measurements of $\mathrm{Lu}$ et al. ${ }^{36}$ clearly demonstrate that the exponential rise of the RDF is suppressed by the charging of particles. The charging introduces a length scale below which clustering of particles is suppressed. They also extended the theoretical framework developed by Chun et al. ${ }^{37}$ to include the "drift" due to Coulomb repulsion force between pair of particles. The functional form of the RDF for charged 
particles as derived by Lu et al. ${ }^{36}$ results from a balance between the outward drift due to Coulomb repulsion and inward drift due to inertia and is as follows:

$$
g(r)=C_{0}\left(\frac{\eta}{r}\right)^{C_{1}} \exp \left[-C_{2} S t_{k}\left(\frac{E_{\text {charge }}}{E_{\text {turb }}}\right)\left(\frac{\eta}{r}\right)^{3}\right] .
$$

$C_{2}$ is defined as

$$
C_{2}=\frac{2}{3 B_{n l}}
$$

where $B_{n l}$ is the dimensionless non-local coefficient for turbulent diffusivity. $E_{\text {charge }}$ and $E_{\text {turb }}$ are the energy of the Coulomb interaction at the Kolmogorov scales and energy of the Kolmogorov eddies, respectively, and defined as

$$
\begin{gathered}
E_{\text {charge }}=\frac{N_{p} q_{p}^{2}}{4 \pi \varepsilon_{0} m_{p} \eta}, \\
E_{\text {turb }}=\left(\frac{\eta}{\tau_{k}}\right)^{2},
\end{gathered}
$$

where $N_{p}$ is the number of particles, $\varepsilon_{0}$ the permittivity of free space, $q_{p}$ and $m_{p}$ are the particle charge and mass, respectively. The limits Lu et al. ${ }^{36}$ chose to apply are also applied here with one exception. The exception is $S t_{k} \ll 1$ : we simulate a range of $S t_{k}$ up to $\sim 40$. Therefore, it should be noted that whilst we use small $S t_{k}$ values to validate our simulation results against Eq. (3), our larger $S t_{k}$ results will not be expected to be accurately predicted by this model of the RDF. This is because the large $S t_{k}$ particles will have a non-local memory of encounters with many turbulent structures. Lu et al. ${ }^{36}$ found that their measurements matched closely with the functional form of RDF above thus providing credence to the drift-diffusion description of preferential concentration. Shaw et al. ${ }^{38}$ have extended the functional form of RDF suggested by Lu et al. ${ }^{36}$ to account for gravitational settling of charged particles. Gravity affects both the uncharged and charged term in the functional of the RDF in a nontrivial way. They measured RDFs for water droplets in homogeneous, isotropic turbulent air.

Here, the attempt is to build both a qualitative and quantitative picture of the effect of gravitational and electrical body forces on particle dispersion. The electrical body force differs in several respects to the gravitational, first, it is a function of the non-homogeneity of the spatial distribution of the particle number density, and also, by extension, it is a function of both space and time. The dependence of preferential concentration on one way coupled turbulent fluctuations, particle inertia, gravity, and bulk charge density are systematically simulated and compared to experimental results. The fluid turbulence is characterised by its Taylor Reynolds number $R e_{\lambda}$. The particle inertia and gravitational settling velocity are characterised by the non-dimensional parameters, $S t_{k}$ and $v_{g}^{*}$. Similarly, the bulk charge density in the domain is characterised by defining a non-dimensional Coulomb velocity, $v_{c}^{*}$.

The remainder of the paper is organized as follows. The governing equations for the fluid and particle phases are presented in Sec. II. In Sec. III, we describe briefly the main features of the pseudo-spectral method employed to directly solve the N-S equations and in Sec. IV the methods used to average and quantify the results obtained. The fluid turbulence characteristics are described in Sec. V and the Lagrangian particle characteristics summarized in Sec. VI. The significance and relevance of the controlling preferential concentration of particles using the electrical body force with and without gravitational interactions is validated against experimental data and further discussed in Sec. VII.

\section{GOVERNING EQUATIONS}

Since the particle-fluid system is one way coupled, the fluid phase is defined by the Eulerian continuity and Navier-Stokes equations, which are defined in a previous publication. ${ }^{28}$ As noted in the Introduction, the non-uniformity of the particle number density, $n_{p}$, generated by preferential 
concentration generates gradients of electric potential ${ }^{39}$

$$
\frac{\partial^{2} \varphi}{\partial x_{i}^{2}}=-\frac{n_{p} q_{p}}{\varepsilon_{0}}, \quad E_{i}=-\frac{\partial \varphi}{\partial x_{i}} .
$$

We are making an implicit assumption here, which is quantified at the start of Sec. VII, in that the length scale used to resolve the spatial gradients of electrical potential are greater than the spacing between the particles. Here, we assume that all forces other than drag and the body forces are negligible. This assumption is based on the large density ratio $\mathrm{O}(3)$ present. We accept that Bassett history effects are comparable under certain conditions ${ }^{6}$ but feel that for this study Eq. (8) is sufficient, and comparable with other benchmark simulations of one-way coupled particle laden turbulence, e.g., ${ }^{4}$

$$
\frac{d U_{i}}{d t}=-\frac{f_{p}}{\tau_{p}}\left(U_{i}-u_{i}^{*}\right)+g_{i}+\frac{q_{p}}{m_{p}} E_{i}^{*}
$$

and $d / d t$ is the derivative with respect to time following the moving particle, $g_{i}$ is the gravitational acceleration, $u_{i}^{*}$ and $E_{i}^{*}$ the instantaneous velocity and electric field of the continuum at the particle location, $U_{i}$ is the instantaneous velocity of the particle. The particle drag function $f_{p}$ (Ref. 40) and particle Reynolds number $\operatorname{Re}_{p}$ are

$$
\begin{aligned}
f_{p} & =1+0.15 \operatorname{Re}_{p}^{0.687}, \\
\operatorname{Re}_{p} & =\frac{\left|U_{i}-u_{i}^{*}\right| \phi_{p}}{v},
\end{aligned}
$$

where $v$ is fluid kinematic viscosity and $\phi_{p}$ the particle diameter. The particle relaxation time is defined as

$$
\tau_{p}=\frac{\phi_{p}^{2}}{18 v} \frac{\rho_{p}}{\rho},
$$

where $\rho_{p}$ and $\rho$ are the particle and fluid densities, respectively.

The gravitational force is characterised by the gravitational settling velocity ${ }^{41}$ and represents the terminal velocity attained by an inertial particle in still fluid,

$$
v_{g}=\tau_{p}\left|g_{i}\right|,
$$

and the corresponding non-dimensional gravitational settling velocity is defined as

$$
v_{g}^{*}=\frac{v_{g}}{u^{\prime}}
$$

where $u^{\prime}$ is the rms turbulence velocity magnitude. In an analogous manner, the relative magnitude of the electric field has been characterised by defining an electrical settling velocity ${ }^{36}$

$$
v_{c}=\tau_{p} \frac{E_{\mathrm{rms}} q_{p}}{m_{p}},
$$

where $E_{\mathrm{rms}}$ is the rms magnitude of electric field in the domain. Effectively, the electrical settling velocity represents the terminal velocity that a particle would attain due to the influence of a specified electric field, in a still fluid. It is important to note that in this case $E_{\mathrm{rms}}$ is generated by spatial perturbations in the particle number density, hence a function of the particle Stokes number $\left(S t_{k}\right)$, bulk number density $\left(n_{p}\right)$, and level of turbulence present $\left(R e_{\lambda}\right)$. The corresponding non-dimensional electrical settling velocity is

$$
v_{c}^{*}=\frac{v_{c}}{u^{\prime}} .
$$

Results are reported from a set of monosized simulations over a range of Taylor Reynolds numbers, Stokes numbers, and non-dimensional gravitational $\left(v_{g}^{*}\right)$ and electrical $\left(v_{c}^{*}\right)$ settling velocities. The Taylor Reynolds numbers reported in this work are 24.2, 45.0, and 80.6. The Stokes numbers 
simulated in this study range from 0.5 to 42 and the non-dimensional gravitational and electrical settling velocities span from 0 to 2.0 and 0 to 2.5 , respectively.

Each of the particles carries a certain amount of charge, $q_{p}$, which is a fraction of the Rayleigh limit $^{42}$ for a droplet of a given size. Drop charge is specified here as $80 \%$ of the Rayleigh limit, reflecting highly charged drops measured experimentally. ${ }^{43}$ For each combination of fluid Taylor Reynolds number and particle Stokes number, the electrical settling velocity is varied to study the effect of the strength of electric field on the particle distribution.

\section{GEOMETRY AND NUMERICAL METHODS EMPLOYED}

Stationary isotropic turbulence is simulated in triply periodic cube of side $2 \pi$ that is one way coupled, unaffected by the particle phase. We use the pseudo-spectral method originally developed by Rogallo. ${ }^{44}$ Evaluation of the nonlinear terms introduces aliasing errors and here these are eliminated using a simple truncation method. ${ }^{45}$ In the present work, flow stationarity has been achieved by adopting the forcing scheme of Eswaran and Pope. ${ }^{46}$ The temporal field is evaluated using a third-order Runge-Kutta scheme. ${ }^{47}$ The time step is calculated according to a predefined Courant Freidricks Levy (CFL) number. All simulations in this study use the CFL number, CFL $=0.75$, and the time step was adjusted dynamically during the simulation. The particle trajectories are calculated using the interpolated physical space velocity and electric fields, and advanced in time using the same third-order Runge-Kutta scheme as used for the fluid phase. To transfer information between variable descriptions, the Eulerian variables are interpolated to the particle position using a thirdorder accurate polynomial ${ }^{48}$ and the Lagrangian information is transferred to the Eulerian control volumes by simple binning. This is required to estimate the number, and hence the charge density in the Eulerian domain, the source term in Eq. (7). Brief details may be found in Scott $e t$ al. $^{28}$ and extended information may be found in $\mathrm{Scott}^{49}$ and Karnik. ${ }^{39}$ Here, Eq. (7) also requires solution and is also obtained through a spectral Fourier method. Since no voltage reference is present in the domain, indeed nor is required, an arbitrary spatial location (control volume) is fixed at zero voltage potential. The electric field generated throughout the domain is obtained with respect to this reference voltage at this location.

\section{AVERAGING AND STATISTICS}

All statistics have been obtained from identical realisations of the turbulent carrier flow at a given Taylor Reynolds number. Identical realisations were achieved by starting all simulations using restart files generated at the end of the fluid initialisation runs where turbulence was proven to have developed to a stationary state. Particles were initialised at random positions within the computational domain and released with initial velocity equal to the local fluid velocity. Both Eulerian and Lagrangian statistics were collected at every time step during the simulation. Instantaneous Lagrangian statistics were ensemble averaged across all particles and are denoted $\bar{\varphi}$. The instantaneous Eulerian statistics were volume-averaged for all nodes in the computational domain and are the form presented unless stated otherwise. An initial period at the start of the simulation (roughly $9 \tau_{E}$ for highest Reynolds number in the simulation) was disregarded to allow the particles to obtain statistically stationary state within the flow. Statistics were then obtained over further $\sim 14 \tau_{E}$.

The $D$ parameter, an Eulerian indicator preferential concentration, is defined as ${ }^{22}$

$$
D=\frac{\sigma-\sigma_{\text {Poisson }}}{\lambda}
$$

where $\sigma$ and $\sigma_{\text {Poisson }}$ represent the standard deviations for the measured particle number density distribution and a Poisson distribution, and $\lambda$ the mean number of particles in the bin.

The RDF, $g(r)$, is calculated by binning particle pairs according to their separation distances and then evaluating the RDF over annular shells of thickness $\Delta$ r. The RDF for a distribution of $N_{p}$ particles is obtained by calculating

$$
g(r)=\frac{P_{r} / \Delta V_{r}}{P / V}
$$


TABLE I. Characteristic turbulence quantities during the stationary period for forced isotropic turbulence.

\begin{tabular}{llccc}
\hline \hline Simulation grid & \multicolumn{1}{c}{$N^{3}$} & $32^{3}$ & $64^{3}$ & $128^{3}$ \\
\hline Taylor micro-scale Reynolds number & $\operatorname{Re}_{\lambda}$ & 24.2 & 45.0 & 80.6 \\
Rms fluctuating velocity & $u^{\prime}$ & 0.827 & 2.560 & 6.241 \\
Energy dissipation rate & $\varepsilon$ & 0.482 & 10.45 & 141.7 \\
Eddy turnover time & $\tau_{E}$ & 1.486 & 0.394 & 0.138 \\
Transverse Taylor micro-scale & $\lambda$ & 0.734 & 0.487 & 0.324 \\
Kolmogorov length scale & $\eta$ & 0.076 & 0.035 & 0.018 \\
Kolmogorov time scale & $\tau_{\eta}$ & 0.230 & 0.049 & 0.013 \\
Longitudinal integral length scale & $L_{11}$ & 1.224 & 1.005 & 0.857 \\
Spatial resolution & $\kappa_{\max } \eta$ & 1.213 & 1.123 & 1.171 \\
\hline \hline
\end{tabular}

where $P_{r}$ is the number of particle pairs separated by a distance $r \pm \Delta r / 2, \Delta V_{r}$ is the volume of the shell of thickness $\Delta r$ located at radius r. $P=N_{p}\left(N_{p}-1\right) / 2$ is the total number of pairs in the simulation and $V=L^{3}$ is the total volume of the domain. With this form of the RDF, a value of unity shows a uniform spacing and values greater than one clustering. The correlation dimension is defined as follows:

$$
D_{2}=\lim _{l \rightarrow 0}(1 / \log l) \log \sum p_{i}^{2},
$$

where $l$ is a variable length scale and $p_{i}$ is the probability that the separation distance between two particles is less than $l$. We compute this quantity by binning all of the particle pairs $N(l)$, according to their separation distance $l$, and calculating the slope of the curve $\log (N(l))$ versus $\log (l)$ over a range of $l$ where a linear dependence exists.

\section{FLUID TURBULENCE CHARACTERISTICS}

In Table I, we list the flow characteristics corresponding to $32^{3}, 64^{3}$, and $128^{3}$ grid configurations, after the one-way coupled flow has become stationary. We defined the fluid density and kinematic viscosity to be $1.0 \mathrm{~kg} / \mathrm{m}^{3}$ and $0.025 \mathrm{~m}^{2} / \mathrm{s}$, respectively. The quantities listed in the table are the rms fluctuating velocity $u^{\prime}$, the longitudinal integral length scale $L_{11}=\frac{\pi}{2 u^{\prime 2}} \int_{0}^{\infty} \frac{E(\kappa)}{\kappa} d \kappa, E(\kappa)$ being the scalar energy spectrum function for wavenumber magnitude $\kappa$, the energy dissipation rate $\varepsilon$, the eddy turnover time $\tau_{E} \equiv L_{11} / u^{\prime}$, the transverse Taylor micro-scale $\lambda$, the Kolmogorov length scale $\eta \equiv\left(v^{3} / \varepsilon\right)^{1 / 4}$, the Kolmogorov time scale $\tau_{\eta} \equiv(v / \varepsilon)^{1 / 2}$, the Taylor Reynolds number $\operatorname{Re}_{\lambda}=u^{\prime} \lambda / v$, and the non-dimensional quantity $\kappa_{\max } \eta$, considered the key factor in ensuring accurate resolution of the velocity field, values near unity showing the Kolmogorov scales are resolved.

\section{LAGRANGIAN SIMULATION CHARACTERISTICS}

Table II lists the particle Stokes numbers and corresponding diameters $\phi_{p}$ and charges $q_{p}$ for the $R e_{\lambda}=24.2,45.0$, and 80.6 charged particle simulations. For all the simulations, $10^{5}$ computational particles are tracked through the domain, the density of all the particles is $1000 \mathrm{~kg} / \mathrm{m}^{3}$ and the surface tension coefficient, $\gamma$ used for all simulations is $0.05 \mathrm{~N} / \mathrm{m} . \varphi_{V}$ provides the particle volume fraction and $\phi_{p} / \eta$ the particle diameter to Kolomogoro length scale ratio. It should be noted that the computational particles are stochastic in nature and each represents an aggregate of $N_{r}$ identical particles. Since in this set of simulations only one way coupling is invoked, the value of $N_{r}$ is adjusted only to obtain a given level of charge density in the domain. Due to spatial fluctuations in this charge density field, a fluctuating electric field is generated which in turn defines the electrical settling velocity defined in Eq. (14).

It is noted that $\phi_{p} / \eta<0.8$ for all the simulations reported in this study and at worst is barely within the point particle assumption limit. The maximum volume fraction of the dispersed phase is 0.13 . We also checked the particle Reynolds number distribution and find they are all $\mathrm{O}(0)$. It is assumed the concentration of particles is small enough such that particle-particle 
TABLE II. Particle diameters $\left(\phi_{p}\right)$, charges $\left(q_{p}\right)$, number of stochastic particles represented by a computational particle $\left(N_{r}\right)$, volume fractions $\left(\varphi_{V}\right)$, and particle diameter to Kolmogorov length scale ratio $\left(\phi_{p} / \eta\right)$ for given Taylor Reynolds and Stokes numbers.

\begin{tabular}{rrrrrrr}
\hline \hline$R e_{\lambda}$ & $S t_{k}$ & $\phi_{p}(\mathrm{~mm})$ & $\mathrm{q}_{\mathrm{p}} \times 10^{9}(\mathrm{C})$ & $\mathrm{N}_{\mathrm{r}}$ & $\varphi_{V}$ & $\phi_{p} / \eta$ \\
\hline 24.2 & 0.5 & 7.2 & 2.89 & 85.8 & $7.86 \times 10^{-5}$ & 0.09 \\
& 1.0 & 10.2 & 4.86 & 51.0 & $2.22 \times 10^{-4}$ & 0.13 \\
& 1.6 & 12.9 & 6.92 & 35.8 & $4.53 \times 10^{-4}$ & 0.17 \\
& 6.4 & 25.8 & 19.58 & 12.7 & $3.63 \times 10^{-3}$ & 0.34 \\
& 12.8 & 36.4 & 32.93 & 7.5 & $1.02 \times 10^{-2}$ & 0.48 \\
45.0 & 25.6 & 51.6 & 55.38 & 4.5 & $2.89 \times 10^{-2}$ & 0.68 \\
& 0.5 & 3.3 & 0.91 & 268.4 & $7.56 \times 10^{-5}$ & 0.08 \\
& 1.0 & 4.7 & 1.53 & 159.6 & $2.14 \times 10^{-5}$ & 0.12 \\
& 2.0 & 6.6 & 2.52 & 94.9 & $6.07 \times 10^{-5}$ & 0.17 \\
& 8.0 & 13.2 & 12.0 & 33.6 & $4.85 \times 10^{-4}$ & 0.33 \\
& 16.0 & 18.6 & 20.18 & 11.9 & $3.84 \times 10^{-3}$ & 0.47 \\
& 32.0 & 26.3 & 0.34 & 900.3 & $9.44 \times 10^{-7}$ & 0.66 \\
& 0.5 & 1.7 & 0.59 & 535.5 & $2.67 \times 10^{-6}$ & 0.13 \\
& 1.0 & 2.5 & 1.11 & 261.4 & $1.15 \times 10^{-5}$ & 0.21 \\
& 2.6 & 3.8 & 3.13 & 92.4 & $9.16 \times 10^{-5}$ & 0.42 \\
& 10.4 & 7.6 & 5.23 & 55.0 & $2.59 \times 10^{-4}$ & 0.59 \\
& 20.8 & 10.7 & 8.78 & 32.7 & $7.33 \times 10^{-4}$ & 0.84 \\
\hline \hline
\end{tabular}

interactions are negligible and the turbulence is not modified by the presence of the particles, though these assumptions are poor for the highest values of volume fraction. However as will be shown, we reveal reasonable agreement with two sources of experimental data, and we believe that reduced real collision probability due to electrical particle pair repulsion enables this lax constraint.

\section{RESULTS AND DISCUSSIONS: PARTICLE-TURBULENCE INTERACTIONS}

The electric field is calculated by taking into account the Eulerian variation of the charge density in the domain, obtained by binning particle charges into control volumes. This method implicitly assumes that the electrical force due to the nearest particle is small compared to the effect of all the particles combined. This assumption is verified by defining an electrical length scale, $x_{E}$, which denotes the separation between particles corresponding to the mean electric field attained in the domain, given by

$$
x_{E}=\frac{q_{p}}{4 \pi \varepsilon_{0} E_{\mathrm{rms}}} .
$$

Figure 1 shows the ratio between the mean minimum particle separation, averaged over all particles and the characteristic Eulerian electrical length scale defined above, for a zero gravity case. The result suggests that the particle separations in the simulations are on average greater than the electrical length scale, $x_{E}$, and as such the electric field at the particle location is well estimated by the use of an Eulerian charge density distribution.

For the results that follow the qualitative results (Figures 2 and 3) and initial quantitative electrical results (Figures 4-7(a)) and without the gravity force. Figure 7(b) compares the normalised mean square displacement for the gravitational body force to the electrical body force given in Figure 7(a). Figures $8(\mathrm{a})$ and 8 (b) show preferential concentration when both gravitational and electrical body forces are present. Finally, Figures 9 and 10 compare specific cases as referenced. ${ }^{36,38}$

The effect of putting charge on particles is evident from Figure 2 where the Coulomb velocity increases from 0 to 1.33 , for the same fluid realization in each case. It is evident that preferential concentration is greater for lower Coulomb velocity level. Figure 3 shows the electric field vectors 


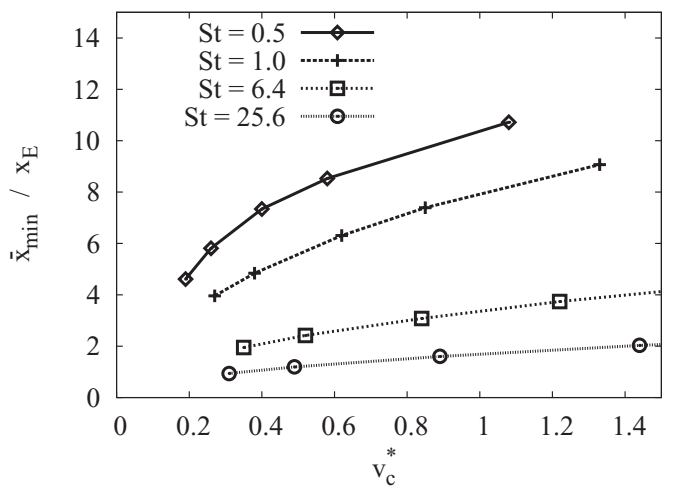

FIG. 1. Variation of ratio of particle separation to electrical length scale with non-dimensional Coulomb velocity at different Stokes numbers for $\operatorname{Re}_{\lambda}=24.2$.

and particle positions for both low and high Coulomb velocity levels. In the case of low charge density (small enough to be unable to homogenise the particle number density in space, large enough to create an electric field), it is seen that the electric field direction points to the centre of the voids in the distribution. The tendency of the electrical body force is to direct the charged particles to regions

(a)

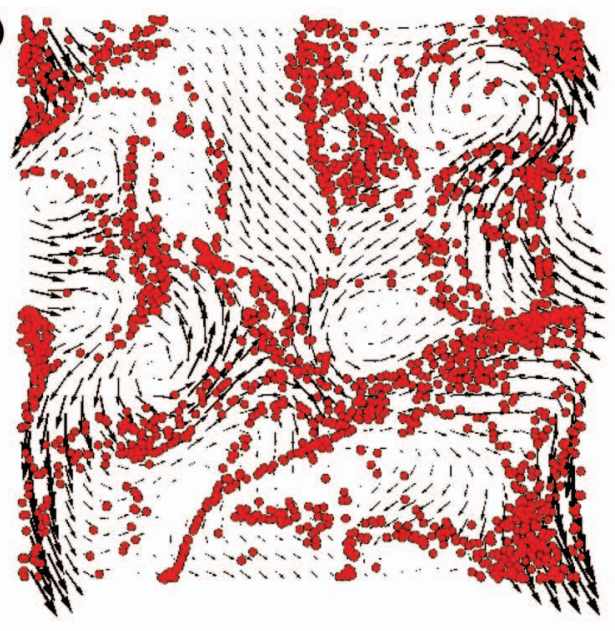

(c)

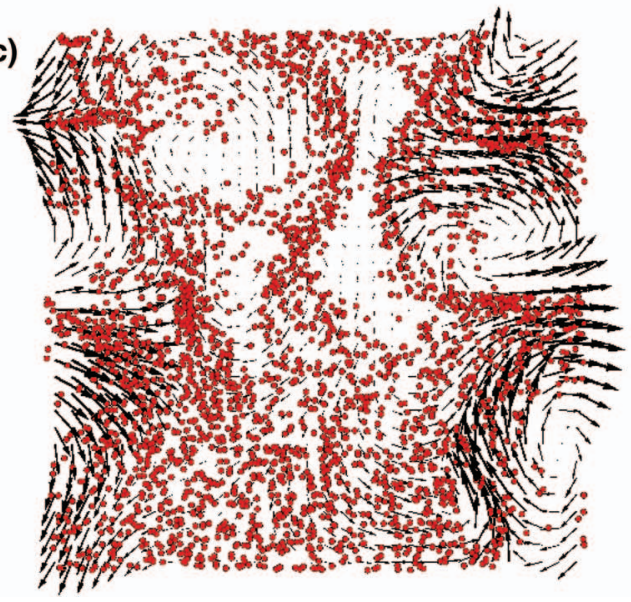

(b)

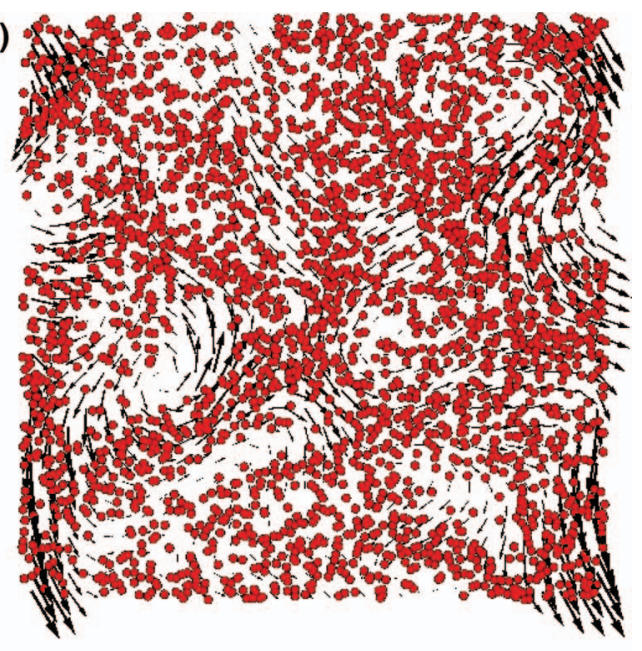

(d)

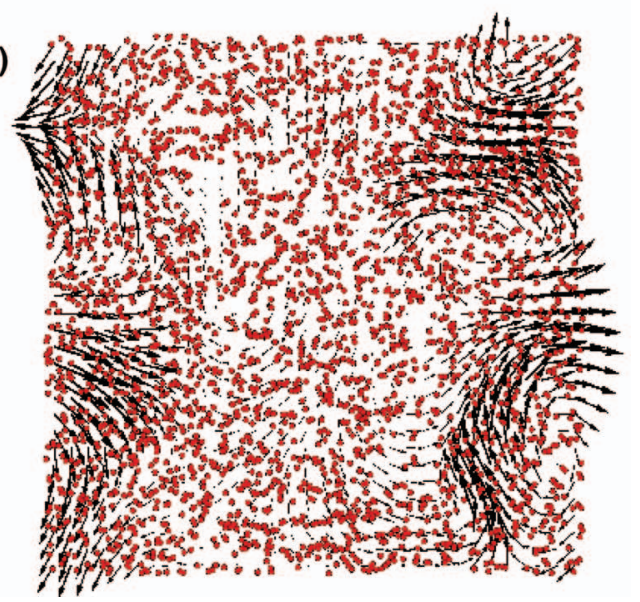

FIG. 2. Particle positions and fluid velocity vectors for $R e_{\lambda}=24.2, S t_{k}=1.0$ and non-dimensional Coulomb velocity, $v_{c}^{*}=0.00(\mathrm{a}), 0.62(\mathrm{~b}), 0.27(\mathrm{c}), 1.33(\mathrm{~d})$. 
(a)

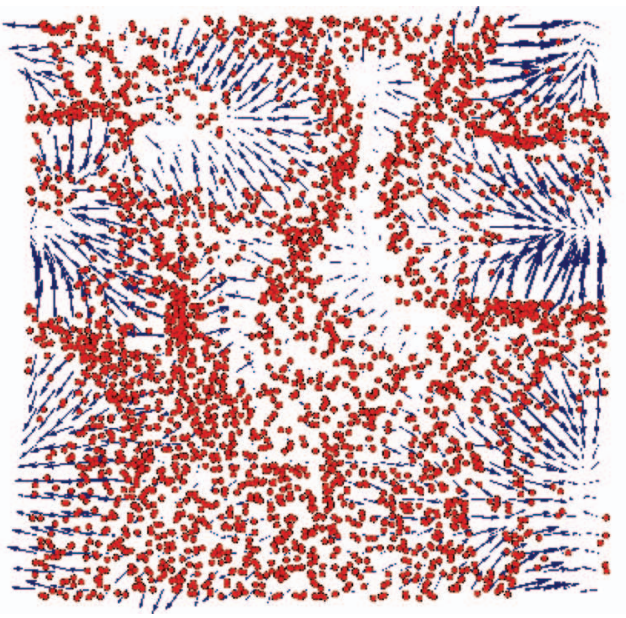

(b)

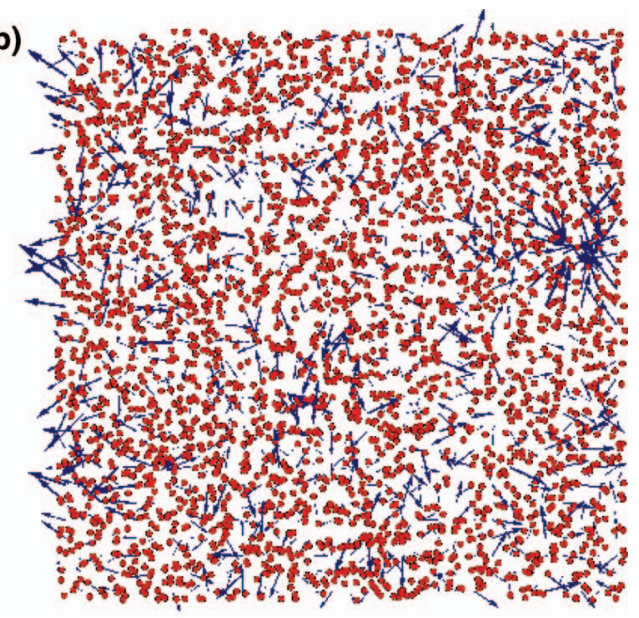

FIG. 3. Electric field vectors and particle positions for $R e_{\lambda}=24.2, S t_{k}=1.0$ and non-dimensional Coulomb velocity, $v_{c}{ }^{*}=0.27$ (a) and $1.33(\mathrm{~b})$.

of low number density. At high Coulomb velocities, a nearly homogeneous distribution of particles is observed and thus the electric field vectors are randomly oriented. Qualitatively, it is observed that Coulomb velocity, $v_{c}{ }^{*} \approx 1.0$ is sufficient to regain homogeneous distribution of particles in the absence of the gravitational body force.

The reduction in preferential concentration with increasing Coulomb velocities is evident from Figure 4 using the $D$ measure, which depends on $\operatorname{Re}_{\lambda}{ }^{28}$ The $D$ values shown in these figures are the peak values when evaluated over a range of bin sizes spanning from the sub-Kolmogorov to the integral scales. It is observed that $v_{c}{ }^{*} \approx 1.0$ is sufficient to mitigate accumulation. This is clearly evident for the high Stokes number particles. At $R e_{\lambda}=45.0,80.6$, the range of non-dimensional Coulomb velocities investigated is limited for low Stokes number particles and thus conclusions can only be formed on the basis of the trend observed in the figures. The trend shows that $v_{c}{ }^{*} \approx 1.0$ would be sufficient to regain a homogenous distribution even at low Stokes numbers.

The radial distribution function is representative of number of particle pairs at a given separation relative to that in the case of uniform distribution of particles. Qualitatively, the RDF is an indicator of particle-particle spacing in clusters of particles. The effect of increasing electric charge on the radial distribution function is seen in Figure 5 for different Reynolds numbers. It is observed that the

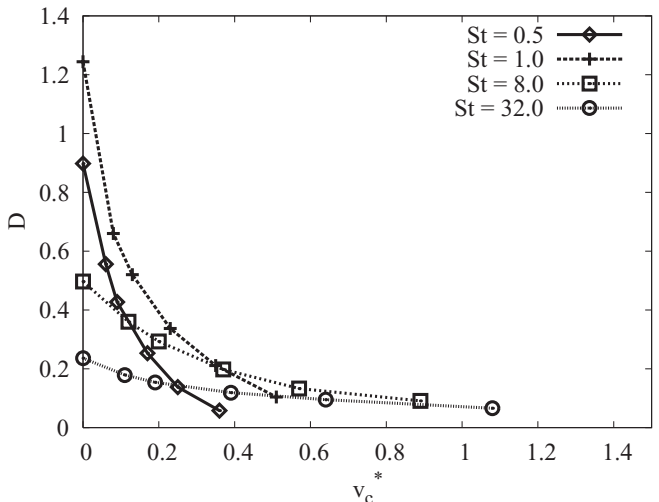

(a)

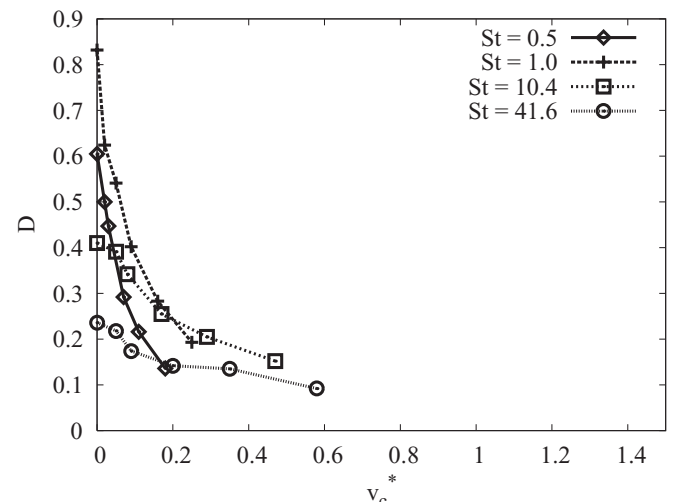

(b)

FIG. 4. Variation of $D$ measure with non-dimensional Coulomb velocity at different Stokes numbers for $R e_{\lambda}=45.0$ (a) and 80.6 (b). (a) Please note $\mathrm{x}$ axis is non-dimensional electrical settling velocity, eps file does not display correctly in automated build. (b) Please note $\mathrm{x}$ axis is non-dimensional electrical settling velocity, eps file does not display correctly in automated build. 


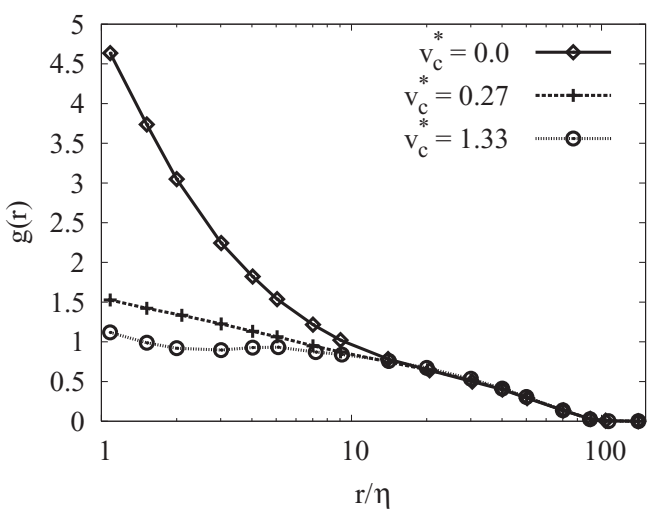

(a)

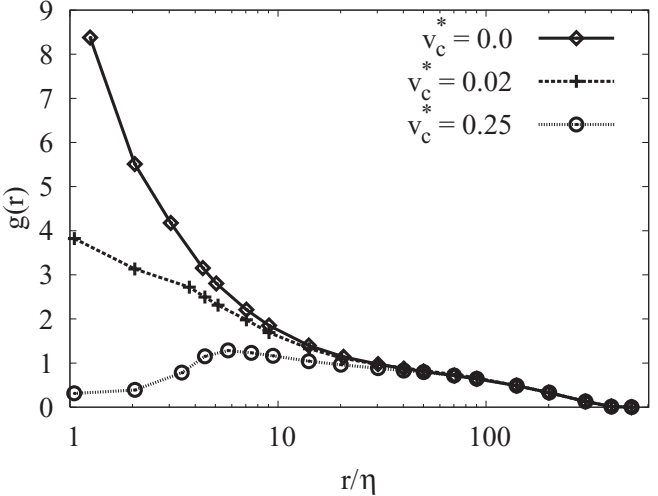

(b)

FIG. 5. Radial distribution functions $(r / \eta>1.0)$ at different non-dimensional Coulomb velocities For $S t_{k}=1.0$ at $R e_{\lambda}$ $=24.2$ (a) and 80.6 (b).

most significant effect of the presence of charges is to reduce the number of pairs with separations up to $10 \eta$. The reduced RDF values imply a reduced collision probability ${ }^{50}$ and this indicates that inclusion of charges on particles not only allows the particles to overcome the centrifugal effects due to turbulent eddies (as evident from the $D$ measure) but charging also diffuses the particle clusters by increasing particle-particle spacing. The data show that the number of particle pairs with separations of the order of the Kolmogorov scale are greatly reduced due to the presence of charge.

In the presence of an electrical force field, the particle distribution is governed by the relative magnitudes of drag and electrical forces acting on the particles and is depicted in Figure 6. Normalised magnitude of electrical force exceeding unity is representative of the electrical forces dominating the particle motion. For $R e_{\lambda}=24.2$, this regime of Lorentz force dominance is seen to take over at $v_{c}{ }^{*}$ $\approx 0.5$, whilst that for $R e_{\lambda}=45.0$ occurs at $v_{c}{ }^{*} \approx 0.4$. It is noted that the range of Coulomb velocities simulated for the highest Reynolds number is limited to non-dimensional Coulomb velocities below 0.4 . Thus, the electrical forces experienced by the $S t_{k}=1.0$ particles at highest Reynolds number are always less than the drag force.

The reduction in dispersion of particles with increasing charge levels, relative to that due to gravitational forces is evident in Figure 7. The reduction in dispersion in the $\mathrm{x}$-direction due to charge at $v_{c}{ }^{*} \approx 1.0$ is seen to be much less than that due to gravity at $v_{g}{ }^{*} \approx 1.0$. In the gravity case, this is due to the crossing trajectories effect and the loss of autocorrelation in directions normal to the gravity (z) direction. It seems also to be the case for the electric body force, which has no preferred direction, yet the rate of dispersion is decreased compared to the gravity case.

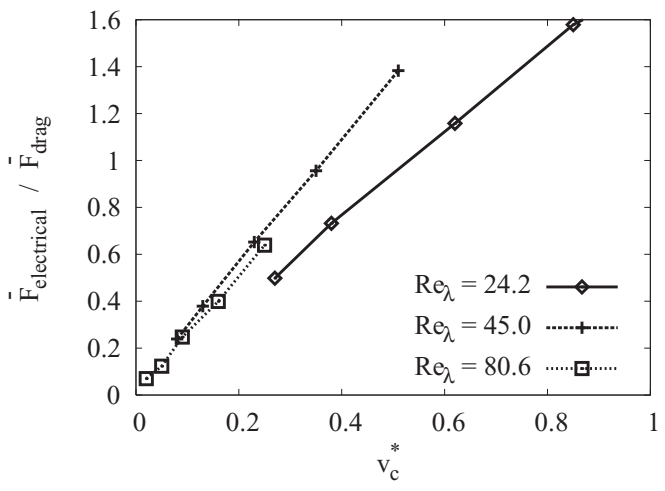

FIG. 6. Electrical force normalised by drag force averaged over all the particles for $S t_{k}=1.0$ particles at different nondimensional Coulomb velocities and Reynolds numbers. 


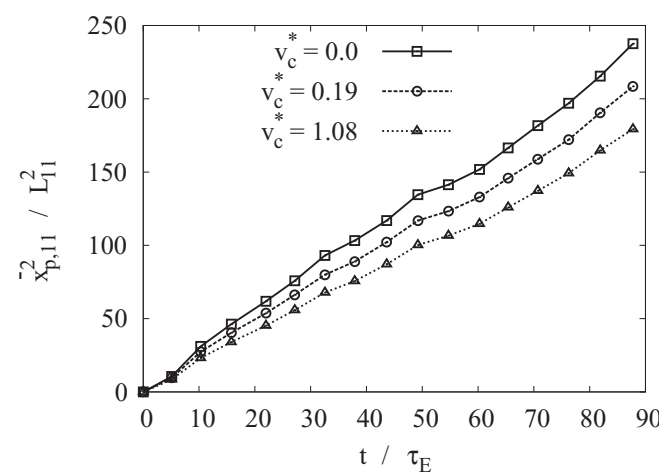

(a)

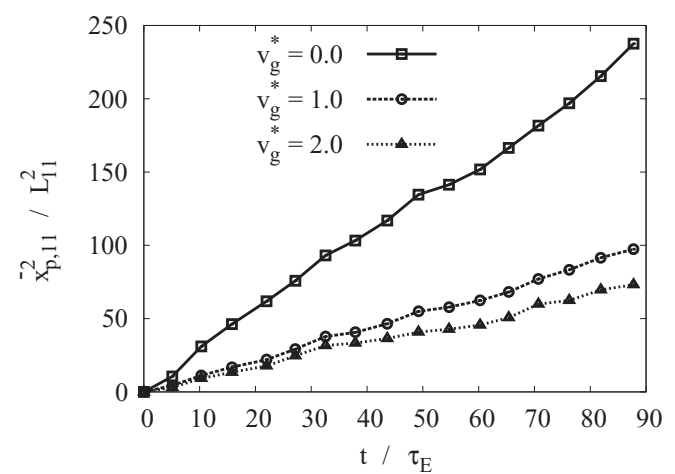

(b)

FIG. 7. Normalised mean square displacement at $R e_{\lambda}=24.2, S t_{k}=0.5$ for different non-dimensional Coulomb velocities (a) and gravitational settling velocities (b).

In order to make the charged particle analysis more practically realistic and enable direct comparison with experimental data, gravitational force is also included in the analysis. The effect of gravity on charged particles with $S t_{k}=1.0$ is explored in Figure 8. Stokes number, $S t_{k}=1.0$ is chosen since effect of gravity is significant at this Stokes number. It is observed that in the presence of gravity, less charge is required to mitigate preferential accumulation. At $v_{g}{ }^{*} \approx 2.0$, a Coulomb velocity corresponding to $v_{c}{ }^{*} \approx 0.6$ is sufficient to homogenise the particle distribution. This is less than the $v_{c}{ }^{*} \approx 1.0$ estimate for homogenising particle distribution in the absence of gravity. A similar improvement (not shown) is found in the particle-particle spacing, characterised by the RDF.

Results from present simulations have also been compared with the experimental results obtained by Lu et al. ${ }^{36}$ and Shaw. ${ }^{38}$ Figure 9 compares the RDF obtained from present study with that reported in the experimental work of Lu et al. ${ }^{36}$ In their experiment, and the simulation test, the charge level in the domain was characterised by the ratio $E_{\text {charge }} / E_{\text {turb }}=2.0, S t_{k}=0.3$, and the $N_{r}$ value in the simulations has been chosen to match the energy ratio reported in the experiment. The nondimensional Coulomb velocity corresponding to the experimental conditions is $v_{c}{ }^{*}=0.035, R e_{\lambda}$ $=80.6$ compared to 84 in the experiment. It should be noted that gravity has been neglected in the result shown in Figure 9 since Lu et al. ${ }^{36}$ made no mention of the influence of gravity in their work. Reasonable but far from excellent agreement is evident. Results from present work have also been compared with experiments on settling charged particles conducted by Shaw et al. ${ }^{38}$ In their experiments, droplets with two sizes were used and subjected to increasing gravitational

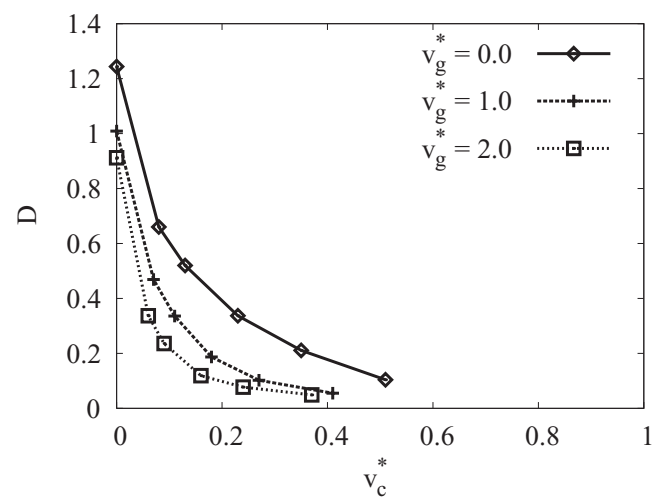

(a)

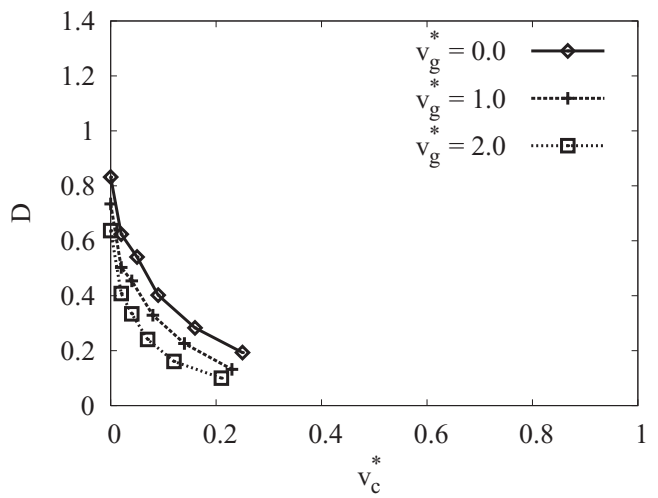

(b)

FIG. 8. Variation of $D$ measure with non-dimensional Coulomb velocity at different gravitational settling velocities for $S t_{k}$ $=1.0$ and $R e_{\lambda}=45.0$ (a) and $R e_{\lambda}=80.6$ (b). 


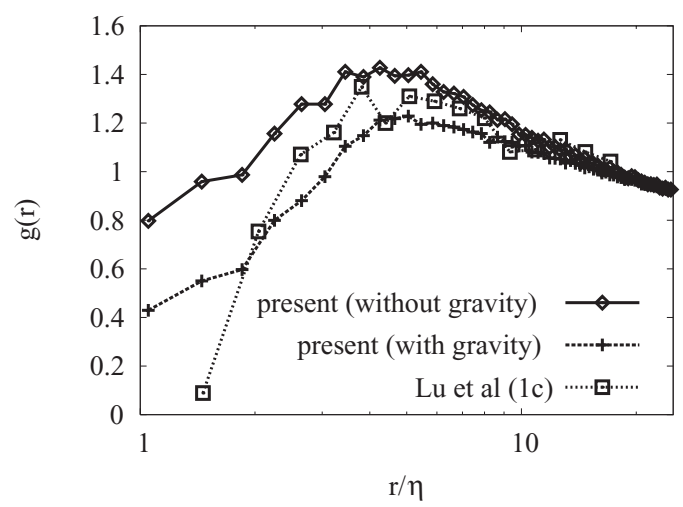

FIG. 9. Radial distribution functions for present simulations compared with that reported in Lu et al. ${ }^{36}$ with similar parameters.

settling using an artificially imposed electric field to create the settling effect. The non-dimensional parameters in the simulations have been adjusted to match the corresponding experimental runs and are listed in Table III.

Figure 10 compares the results from present simulations with the "doublet" experimental results reported in Shaw et al., ${ }^{38}$ case 3d of Table III, the highest level of Coulomb velocity. Reasonably good agreement with experiments is observed given the uncertainties involved in the experiments. This agreement lends credence to the analysis of settling charged particles carried out in present work. It can be noted that the non-dimensional Coulomb velocities for the experiments are very small, whereas the current analysis shows that $v_{c}{ }^{*} \approx 1.0$ tends to appreciably mitigate preferential concentration. It is also noted that the results from simulations match the functional form of the RDF for settling charged particles proposed by Shaw et al. ${ }^{38}$ However, it should be borne in mind that the theory is strictly valid only for $S t_{k}=1$ and interpretation of present results at high Stokes numbers using the theory is strictly not permissible.

The estimated non-dimensional Coulomb velocity to mitigate accumulation, $v_{c}{ }^{*} \approx 1.0$, corresponds to bulk charge density levels in the domain, $Q_{0}$, of $\sim 20-80 \mu \mathrm{C} / \mathrm{m}^{3}$. Here, we estimate the bulk charge density levels prevalent sprays generated by practical charged injection atomizer. The charged round liquid jet emerging from the atomizer, at $\mathrm{z}=0$, is initially a cylinder of diameter $\mathrm{d}_{0}$ and volume charge density, $Q_{0}$. The spray plume then expands as a cone, entraining air and reducing the bulk charge. For a spray half-angle $\theta$, the spray radius at any section $z$, is given by, $r=\mathrm{d}_{0} / 2$ $+\mathrm{z} \tan \theta$ and assuming velocity of the plume does not change, then the volume charge density at an axial displacement $\mathrm{z}$ from the atomizer orifice is a function of the cross-sectional area. The volume

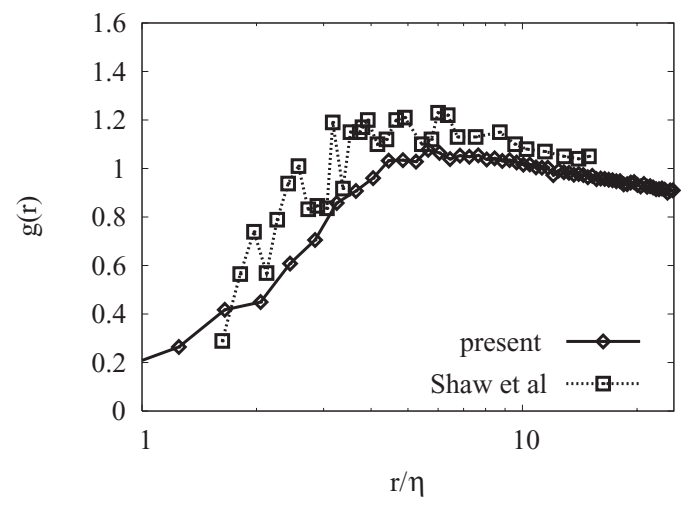

FIG. 10. Radial distribution functions for present study compared with that reported in Shaw et al. ${ }^{38}$ for Run $3 \mathrm{~d}$ (refer Table III). 
TABLE III. Non-dimensional parameters for present simulations corresponding to experimental conditions reported by Shaw et al. ${ }^{30}$ Simulations $1 \mathrm{~s}, 2 \mathrm{~s}, 3 \mathrm{~s}$ correspond to the "singlet" experimental runs at increasing gravity levels. Simulations $1 \mathrm{~d}, 2 \mathrm{~d}$, $3 \mathrm{~d}$ correspond to the "doublet" experimental runs at increasing gravity levels.

\begin{tabular}{lcccccc}
\hline \hline & $1 \mathrm{~s}$ & $2 \mathrm{~s}$ & $3 \mathrm{~s}$ & $1 \mathrm{~d}$ & $2 \mathrm{~d}$ & $3 \mathrm{~d}$ \\
\hline$R e_{\lambda}$ & 80 & 80 & 80 & 80 & 80 & 80 \\
$S t_{k}$ & 0.14 & 0.14 & 0.14 & 0.22 & 0.22 & 0.22 \\
$v_{g}{ }^{*}$ & 0.354 & 0.664 & 0.797 & 0.554 & 1.041 & 1.262 \\
$v_{c}{ }^{*}$ & 0.0067 & 0.0070 & 0.0068 & 0.022 & 0.021 & 0.021 \\
\hline \hline
\end{tabular}

charge density at any section $\mathrm{z}$ is then given by, $Q_{0}(\mathrm{z})=Q_{0} /\left(1+4 \mathrm{z} \tan \theta / \mathrm{d}_{0}+4 \mathrm{z}^{2} \tan ^{2} \theta / \mathrm{d}^{2}{ }_{0}\right)$. For a spray with initial diameter, $\mathrm{d}_{0}=150 \mu \mathrm{m}$, initial charge density, $Q_{0}=4.0 \mathrm{C} / \mathrm{m}^{3}$ and spray angle of $10^{\circ}$, the charge levels found in this study $\left(20-80 \mu \mathrm{C} / \mathrm{m}^{3}\right)$ correspond to maximum displacement $\sim 10 \mathrm{~cm}$ from the nozzle tip. The assumption of constant velocity is rather poor, since in reality the spray plume will decelerate and the downstream particle concentration will increase. Therefore, the estimates given here of bulk specific charge (spray + air) can be considered conservative. It is seen that the levels of charge required to significantly reduce the preferential accumulation are within those capable of charged injection atomization devices currently available. In practical atomizers, the first non-homogeneous clustering of particles tends to occur in the first few centimetres from the nozzle. Thus, placing charges on particles holds the promise of creating a homogeneous mixture downstream of the spray. This is potentially useful in the context of combustion where demixing of the fuel-vapour mixture results in incomplete combustion and formation of soot and other pollutants.

\section{CONCLUSIONS}

Charging of particles has been proposed as a means of mitigating preferential concentration. Results from simulations of charged particles in isotropic turbulence have been presented both neglecting and including effect of gravity. The findings can be summarised as follows.

In the absence of gravity, it is estimated that $v_{c}{ }^{*} \approx 1.0$ is sufficient to homogenise a preferentially accumulated particle distribution. It is seen that charging drastically reduces the RDF values at subKolmogorov scale separations. This implies that charging the particles is an efficient means to destroy particle clusters.

The presence of charges reduces the dispersion of particles. The reduction in dispersion due to presence of charge on particles at $v_{c}{ }^{*} \approx 1.0$ is much less than the reduction due to gravity at $v_{g}{ }^{*}$ $\approx 1.0$.

On incorporating the gravitational force, the amount of charge required to homogenise the particle distribution is reduced. It is estimated that $v_{c}{ }^{*} \approx 0.6$ is sufficient to homogenise particle distribution at $v_{g}{ }^{*} \approx 1.0$. This estimation is corroborated by several different indicators of preferential concentration.

The results from simulations carried out in present work agree reasonably well with corresponding experiments reported in literature. The RDF obtained from simulations matches the functional form of RDF proposed initially by Chun et al. ${ }^{37}$ and the extended form for settling charged particles suggested by Shaw et al. ${ }^{38}$ Thus, the drift-diffusion model of Chun et al. ${ }^{39}$ seems to be a fundamentally correct starting point to explain the clustering of inertial particles.

It is shown that the bulk charge density levels required to homogenise particle distribution correspond to those attained around $10 \mathrm{~cm}$ from tip of the nozzle in practical charged injection atomizers. Thus, placing charges on particles holds the promise of creating a homogeneous mixture downstream of the spray. This is especially useful in the context of combustion where demixing of the fuel-vapour mixture results in incomplete combustion and formation of soot and other pollutants. 
${ }^{1}$ M. I. Yudine, "Physical considerations on heavy-particle diffusion. Atmospheric diffusion and air pollution," Adv. Geophys. 6, 185-191 (1959).

${ }^{2}$ G. T. Csanady, "Turbulent diffusion of heavy particles in the atmosphere," J. Atmos. Sci. 20, 201-208 (1963).

${ }^{3}$ W. W. Reeks, "On the dispersion of small particles suspended in an isotropic turbulent fluid," J. Fluid Mech. 83(3), 529-546 (1977).

${ }^{4}$ L. P. Wang and M. R. Maxey, "Settling velocity and concentration distribution of heavy-particles in homogeneous isotropic turbulence," J. Fluid Mech. 256, 27-68 (1993).

${ }^{5}$ J. J. Riley and G. S. Patterson, "Diffusion experiments with numerically integrated isotropic turbulence," Phys. Fluids 17(2), 292-297 (1974).

${ }^{6} \mathrm{~S}$. Elghobashi and G. C. Truesdell, "Direct simulation of particle dispersion in a decaying isotropic turbulence," J. Fluid Mech. 242, 655-700 (1992).

${ }^{7}$ W. H. Snyder and J. L. Lumley, "Some measurements of particle velocity autocorrelation functions in a turbulent flow," J. Fluid Mech. 48(1), 41-71 (1971).

${ }^{8}$ J. K. Eaton and J. R. Fessler, "Preferential concentration of particles by turbulence," Int. J. Multiphase Flow 20, 169-209 (1994).

${ }^{9}$ K. D. Squires and J. K. Eaton, "Preferential concentration of particles by turbulence," Phys. Fluids A 3(5), 1169-1179 (1991).

${ }^{10}$ H. Yoshimoto and S. Goto, "Self-similar clustering of inertial particles in homogeneous turbulence," J. Fluid Mech. 577, 275-286 (2007).

${ }^{11}$ G. Boffetta, F. De Lillo, and A. Gamba, "Large scale inhomogeneity of inertial particles in turbulent flows," Phys. Fluids 16(4), L20-L23 (2004).

${ }^{12} \mathrm{~S}$. Goto and J. C. Vassilicos, "Self-similar clustering of inertial particles and zero-acceleration points in fully developed two-dimensional turbulence," Phys. Fluids 18, 115103 (2006).

${ }^{13}$ S. Goto and J. C. Vassilicos, "Sweep-stick mechanism of heavy particle clustering in fluid turbulence," Phys. Rev. Lett. 100, 054503 (2008).

${ }^{14}$ E. Balkovsky, G. Falkovich, and A. Fouxon, "Intermittent distribution of inertial particles in turbulent flows," Phys. Rev. Lett. 86, 2790-2793 (2001)

${ }^{15}$ J. C. Sommerer and E. Ott, "Particles floating on a moving fluid: a dynamically comprehensible physical fractal," Science 259, 335-339 (1993).

${ }^{16} \mathrm{~J}$. Bec, "Multifractal concentrations of inertial particles in smooth random flow," J. Fluid Mech. 528, 255-277 (2005).

${ }^{17}$ M. Wilkinson, B. Mehlig, S. Ostlund, and K. P. Duncan, "Unmixing in random flows," Phys. Fluids 19, 113303 (2007).

${ }^{18}$ A. N. Osiptov, "Lagrangian modelling of dust admixture in gas flows," Astrophys. Space Sci. 274, 377-386 (2000).

${ }^{19}$ R. H. A. Ijzermans, E. Meneguz, and M. W. Reeks, "Segregation of particles in incompressible random flows: singularities, intermittency and random uncorrelated motion (RUM)," J. Fluid Mech. 653, 99-136 (2010).

${ }^{20} \mathrm{E}$. Meneguz and M. W. Reeks, "Statistical properties of particle segregation in homogeneous isotropic turbulence," J. Fluid Mech. 686, 338-351 (2011)

${ }^{21}$ P. Fevrier, O. Simonin, and K. D. Squires, "Partitioning of particle velocity in gas-solid turbulent flows into a continuous field and a spatially uncorrelated random distribution: theoretical formalism and numerical study," J. Fluid Mech. 553, $1-46$ (2005).

22 J. R. Fessler, J. D. Kulick, and J. K. Eaton, "Preferential concentration of heavy-particles in a turbulent channel flow," Phys. Fluids 6(11), 3742-3749 (1994).

${ }^{23}$ A. Aliseda, A. Cartellier, F. Hainaux, and J. C. Lasheras, "Effect of preferential concentration on the settling velocity of heavy particles in homogeneous isotropic turbulence," J. Fluid Mech. 468, 77-105 (2002).

${ }^{24}$ S. Sundaram and L. R. Collins, "Collison statistics in an isotropic particle-laden turbulent suspension. Part 1. Direct numerical simulations,” J. Fluid Mech. 335, 75-109 (1997).

${ }^{25}$ L. R. Collins and A. Keswani, "Reynolds number scaling of particle clustering in turbulent aerosols," New J. Phys. 6, 119 (2004).

${ }^{26}$ J. Bec, L. Biferale, M. Cencini, A. Lanotte, S. Musacchio, and F. Toschi, "Heavy particle concentration in turbulence at dissipative and inertial scales," Phys. Rev. Lett. 98, 084502 (2007).

${ }^{27}$ M. Van Aartrijk and H. J. H. Clercx, "Preferential concentration of heavy particles in stably stratified turbulence," Phys. Rev. Lett. 100, 254501 (2008).

${ }^{28}$ S. J. Scott, A. U. Karnik, and J. S. Shrimpton, “On the quantification of preferential accumulation,” Int. J. Heat Fluid Flow 30, 789-795 (2009).

${ }^{29}$ G. D. Roy, S. M. Frolov, A. A. Borisov, and D. W. Netzer, "Pulse detonation propulsion: challenges, current status, and future perspective," Prog. Energy Combust. Sci. 30, 545-672 (2004).

${ }^{30}$ M. Yao, Z. Zheng, and H. Liu, "Progress and recent trends in homegeous charge compression ignition (HCCI) engines," Prog. Energy Combust. Sci. 35(5), 398-437 (2009).

${ }^{31}$ J. S. Shrimpton and A. J. Yule, "Characterisation of charged hydrocarbon sprays for application in combustion systems," Exp. Fluids 26(5), 460-469 (1999).

${ }^{32}$ A. R. H. Rigit and J. S. Shrimpton, "Estimation of the diameter-charge distribution in polydispersed electrically charged sprays of electrically insulating liquids," Exp. Fluids 46(6), 1159-1171 (2009).

${ }^{33}$ J. Bellan, "A new approach to soot control in diesel-engines by fuel-drop charging," Combust. Flame 51(1), 117-119 (1983).

${ }^{34}$ J. Bellan and K. Harstad, "Electrostatic dispersion and evaporation of clusters of drops of high-energy fuel for soot control," in Proceedings of the Symposium (International) on Combustion, Italy (Elsevier, Amsterdam, 1996), Vol. 26(1), pp. 1713-1722. 
${ }^{35}$ V. M. Alipchenkov, L. I. Zaichik, and O. F. Petrov, "Clustering of charged particles in isotropic turbulence,” High Temp. 42(6), 919-927 (2004).

${ }^{36}$ J. Lu, H. Nordsiek, E. W. Saw, and R. A. Shaw, "Clustering of charged inertial particles in turbulence," Phys. Rev. Lett. 104(18), 184505 (2010).

${ }^{37}$ J. H. Chun, D. L. Koch, S. L. Rani, A. Ahluwalia, and L. R. Collins, "Clustering of aerosol particles in isotropic turbulence," J. Fluid Mech. 536, 219-251 (2005).

${ }^{38}$ R. A. Shaw, J. A. Lu, and H. Nordsiek, "Clustering of settling charged particles in turbulence: theory and experiments," New J. Phys. 12, 123030 (2010).

${ }^{39}$ A. U. Karnik, "Direct numerical investigations of dilute dispersed flows in homogeneous turbulence," Ph.D. dissertation, University of Southampton, United Kingdom, 2011.

${ }^{40}$ A. Putnam, "Integratable form of the drop drag coefficient," ARS J. 31, 1467 (1961).

${ }^{41}$ M. R. Maxey, "The gravitational settling of aerosol-particles in homogeneous turbulence and random flow-fields," J. Fluid Mech. 174, 441-465 (1987).

${ }^{42}$ Lord Rayleigh, "On the equilibrium of liquid conducting masses charged with electricity," Philos. Mag. 5(14), 184-186 (1882).

${ }^{43}$ D. C. Taflin, T. L. Ward, and E. J. Davis, "Electrified droplet fission and the Rayleigh limit," Langmuir 5, 376-384 (1989).

${ }^{44}$ R. S. Rogallo, "Numerical experiments in homogenous turbulence," Technical Memorandum NASA-TM-81315, NASA, 1981.

${ }^{45}$ C. Canuto, M. Y. Hussaini, A. Quarteroni, and T. A. Zang, Spectral Methods in Fluid Dynamics, Springer Series in Computational Physics (Springer, Berlin, 1988).

${ }^{46}$ V. Eswaran and S. B. Pope, "An examination of forcing in direct numerical simulations of turbulence," Comput. Fluids 16(3), 257-278 (1987).

${ }^{47}$ J. H. Williamson, “Low storage Runge-Kutta schemes," J. Comput. Phys. 35, 48-56 (1980).

${ }^{48}$ J. Ferziger and M. Peric, Computational Methods for Fluid Dynamics (Springer-Verlag, Berlin, 1996).

${ }^{49}$ S. J. Scott, "PDF based method for modelling polysized particle laden turbulent flows without size class discretisation," Ph.D. dissertation, Imperial College London, 2006.

${ }^{50}$ W. C. Reade and L. R. Collins, "Effect of preferential concentration on turbulent collision rates," Phys. Fluids 12(10), 2530-2540 (2000) 